\title{
Software-Based Mass Spectral Enhancement to Remove Interferences from Spectra of Unknowns
}

\author{
Nelson R. Herron and Joseph R. Donnelly \\ Lockheed Environmental Systems and Technologies Company, Las Vegas, Nevada, US $\Lambda$ \\ G. Wayne Sovocool \\ U.S. Environmental Protection Agency, National Exposure Research Laboratory, Characterization Research \\ Division, Las Vegas, Nevada, USA
}

Gas chromatography -mass spectrometry data from the analysis of complex environmental samples were converted into ASCII text and imported into a personal computer spreadsheet. A macro was written to perform mass spectral enhancement by statistical and mathematical procedures to separate the spectra of compounds of interest from interfering mass spectral responses, such as those of broadly eluting hydrocarbons. The extracted mass spectra were compared to reference spectra, with the result that usually $80-90 \%$ of the ions common to those in the reference spectra were successfully extracted by using this method. This procedure improved mass spectral quality and the ability of the data system to perform successful library searches. The fitted quality parameters showed systematic improvements after the data were subjected to the spectral enhancement procedures. These procedures could help to identify unknowns by separating their spectra from other signals, such as those of background aliphatic hydrocarbons. (J Am Soc Mass Spectrom 1996, 7, 598-604)

$\mathrm{M}$ ost environmental samples are analyzed either by a general protocol suitable for a suite cof target analytes or by methods that were designed for subsets of analytes [1,2]. Bccause these methods are not optimized for all analytes or matrices to which they are applied, it is sometimes necessary to reextract environmental samples by using different procedures, to perform alternate cleanup methods on the extracts, or to use highly specialized instrumentation to remove interferences and separate some analytes of interest from contaminants. Development and implementation of these procedures are time consuming and costly. In addition, reanalysis is possible only if sufficient samples or extracts are available.

One option for the user of data from the gas chromatography-mass spectrometry (GC/MS) analysis of complex samples such as environmental extracts is the use of computer software and related studies of the data to identify unknowns instead of conducting further analyses of the samples. The capabilities of modern personal computers make it worthwhile to consider the use of mathematical methods for spectral enhancement and background subtraction to isolate the spectra of unknown compounds of interest from

Address reprint requests to Dr. Joseph R. Donnelly, I ockheed Environmental Systems and Technologies Company, 980 Kelly Johnson Drive, Las Vegas, NV 89119 interferences. This is especially evident when there are large numbers of samples present from a site that contains numerous interfering compounds.

A potentially powerful method of mass spectral enhancement via background rejection could result if a concept proposed by Colby [3] is employed. This mass spectral enhancement or "spectral deconvolution" approach is functionally related to the Biller-Biemann spectral isolation algorithm [4] wherein the data system searches the mass chromatogram and flags mass spectra for which a selected number of masses (c.g., three) maximize within a set number of scans (e.g., two). An enhancement routine then discards ions that do not maximize within that retention time window. the implementation of this concept includes features that resemble a digital implementation of the widely used phase-locked amplifier in electronics. This approach would be valuable for the identification of unknowns in environmental and other complex samples.

As a part of ongoing research on tentatively identified compounds (TICs), the U.S. Environmental Protection Agency, National Exposure Research Laboratory, Characterization Research Division-Las Vegas has studied unknown compounds reported in Superfund sample data submitted by the Contract Laboratory Program (CLP) [1]. For this study, the above-noted concept proposed by Colby was investigated and im- 
plemented through a commercially available personal computer spreadsheet. For test data, environmental sample gas chromatography-mass spectrometry (GC/MS) raw data tapes were utilized from the CLP.

\section{Experimental}

The spectral enhancement approach was designed and implemented as a set of macros written in Visual Basic for Applications in Microsoft EXCEL ${ }^{\mathrm{TM}}$ (Microsoft Corp., Redmond, WA). (The macro is available from the authors upon request with a 3.5-in. diskette in DOS format.) Raw CLP GC/MS data stored on tapes were accessed from a Hewlett-Packard (HP; Palo Alto, CA) data system and the National Institute of Standards and Technology (NIST, Gaithersburg, MD) mass spectral data base. The data files were converted to ASCII text with MassTransit ${ }^{\mathrm{TM}}$ Version 1.02a (Palisade Corp., Newfield, NY, 1993) and imported into EXCEL ${ }^{\mathrm{IM}}$ Version 5.0 (Microsoft Corp., 1994). These data files were subsequently manipulated by using the above-noted macro (see Table 1 for a block diagram of the functions performed by this macro) to perform the sorting and the statistical and mathematical procedures necessary to separate the selected peaks from interfering compounds. The intensity maxima (peaks) for each ion in a mass chromatogram were determined and their raw retention times (scan numbers) were noted. The raw scan numbers were adjusted to fractional scan numbers for each mass-to-charge ratio to match the precise time during a scan when that mass was measured. This adjustment was expressed as an offset term $O_{S}$,

Table 1. The steps performed in the mass spectral enhancement operation

\begin{tabular}{|c|c|}
\hline Step & Action \\
\hline 1 & Convert the raw data into text format \\
\hline 2 & $\begin{array}{l}\text { Parse data by using EXCEL } \\
\text { two-dimensional matrix that places the reported } \\
\text { intensity for each ion present in a cell indexed } \\
\text { to the mass and scan numbers }\end{array}$ \\
\hline 3 & $\begin{array}{l}\text { Start a sliding window to look for all ions that } \\
\text { are present in three successive scans and iden- } \\
\text { tify each case where the ion maximizes in the } \\
\text { center of the window. }\end{array}$ \\
\hline 4 & $\begin{array}{l}\text { Adjust the nominal scan time for time lag due to } \\
\text { instrument scanning } \mathrm{O}_{S}\end{array}$ \\
\hline 5 & $\begin{array}{l}\text { Adjust the peak retention to the observed ion } \\
\text { distribution by using quadratic fit } O_{A}\end{array}$ \\
\hline 6 & $\begin{array}{l}\text { Place the time-corrected data in a new matrix } \\
\text { and sort with respect to the adjusted retention } \\
\text { time }\end{array}$ \\
\hline 7 & $\begin{array}{l}\text { Apply a filter to group ions based on adjusted } \\
\text { retention times and generate an enhanced mass } \\
\text { chromatogram }\end{array}$ \\
\hline 8 & $\begin{array}{l}\text { Extract ions within a given time range (peak) to } \\
\text { producc a mass spectrum with enhanced back- } \\
\text { ground rejection }\end{array}$ \\
\hline
\end{tabular}

which was added to the scan number and is defined as follows:

$$
O_{\mathrm{S}}=\left(M_{\text {curr }}-M_{\min }\right) /\left(M_{\max }-M_{\min }\right)
$$

where $M_{\text {curr }}$ is the current mass, $M_{\min }$ is the minimum mass sampled (usually $m / z$ 35), and $M_{\max }$ is the maximum mass sampled (usually $m / z$ 500). 'The numerator represents how far into the scan the mass occurred and the denominator represents the full scan range. Hence, for linear scanning, $O_{S}$ represents the simple fraction of the full scan completed at the given mass. This approach assumes linear mass scanning from low mass to high mass and ignores the small $(\sim 1-3 \%)$ systematic error introduced by resetting the quadrupole scan voltage. As defined, $O_{S}$ is always nonnegative and less than 1 ; it is therefore an intrascan correction.

Near the apex of a mass chromatographic peak, the peak generally appears to be reasonably parabolic and can be represented by the following quadratic equation:

$$
Y=a X^{2}+b X+c
$$

where $Y$ is the intensity in arbitrary data system units, $\mathrm{X}$ is the true retention time (in scan numbers), and $a$, $b$, and $c$ are constants to be determined. The quadratic form was fitted exactly to each peak ion intensity and the intensities of the preceding and succeeding scans. By selecting these three intensities, the three coefficients could be determined exactly.

The retention time of the apex of the fitted curve was expected to be an accurate estimation of the true retention time of the conslituent of interest, within constraints imposed by signal noise from scan to scan. This apex was found readily by using the common technique that takes the first derivative of the quadratic function and sets it equal to zero, as follows:

$$
Y^{\prime}=2 a X+b=0
$$

where $Y^{\prime}$ is the first derivative.

A variety of methods, which include packaged optimization routines in EXCEL ${ }^{\mathrm{TM}}$, were available to solve the preceding algebraic equations for the true retention time $X$. A fast, extremely simple axis-translation solution to the problem was selected that reflected the simplicity of the mass chromatographic data in a spreadsheet format. A peak intensity $I_{0}$, presented in scan coordinates in the spreadsheet, was the origin for the fitted quadratic equation, and each of the adjoining intensities was set to coordinates of 1 and -1 , respectively, with values of $I_{\text {R(ight) }}$ and $I_{L(\text { efft })}$. These changes of variable provided a simple closed form for the offset of the apex $O_{A}$, in scan coordinates:

$$
\mathrm{O}_{\mathrm{A}}=-\left(I_{\mathrm{K}}-I_{\mathrm{L}}\right) / 2\left(I_{\mathrm{R}}+I_{\mathrm{L}}-2 I_{0}\right)
$$

$O_{A}$ ranged from -0.5 to +0.5 and was therefore an interscan correction. The optimized retention time for the mass was obtained by addition of $O_{S}$ and $O_{A}$ to the raw scan number of the peak. Once a peak was identified, the entire optimization procedure was per- 
formed compactly by a single line of code in the macro that contained only one multiplication and two division operations (steps 4 and 5 in Table 1). Minimization of multiplication and division operations was desired because these operations were more timeintensive for the computer than were additions and subtractions.

As an example of this procedure, consider $m / z 50$ at scans 828,829 , and 830 with intensities of 841,2467 , and 1536, respectively. Calculations provided $\mathrm{O}_{S}=$ 0.0323 scan, $O_{\mathrm{A}}-0.1359$ scan, and an optimized retention time that corresponded to scan 829.1682.

The quadratically optimized retention times, masses, and observed peak intensities from the mass chromatograms were stored in a list as they were calculated. After all of the peaks were optimized, the list was sorted with respect to retention time. The intensities of all masses that fall within selected sequential retention time windows (summing intervals) over the entire chromatographic time range were summed and placed into a second list of retention times and intensities. For example, a summing interval of 0.2 scan would result in the summation and collection of all masses of peaks that have intensity optimizations that occur between scan 1000.0 and 1000.2 into a list for the total ion current signal of that compound. The summing interval duration was set at the beginning of the experiment and was typically between 0.1 and 0.33 scans. The selection of a summing interval shorter than one scan yielded a total ion current chromatogram with greatly enhanced resolution, which showed distinct baseline-resolved peaks under the high levels of background signals. The mass spectra obtained for these mathematically resolved peaks were free of most background mass responses because few of the background masses maximized coincidentally with the masses of the peaks under investigation.

After the spectral enhancement procedure was completed, a peak of potential interest was selected to give the background-rejected mass spectrum. The mass spectrum was exported with the appropriate header to a text file. This file was imported into a mass spectrometer data system through a program such as MassTransit, and the mass spectrum was searched against a reference data base such as the NIST mass spectral library. Table 1 outlines the steps required to perform a spectral enhancement analysis of a mass chromatogram.

\section{Results and Discussion}

The original report on mass spectral enhancement and background signal rejection techniques demonstrated their potential on solutions of known reference standards [3]. In the present study, subject data files on actual environmental sample analyses were translated into a format suitable for a personal computer spreadsheet. Spectral isolation or enhancement was performed in 5-10 min (e.g., a 250-scan data set for the analysis of a set of standards took $7 \mathrm{~min}$ on an 80486 66-MHz computer, Gateway 2000, N. Sioux City, SD). This time requirement is far less than the hours of time necessary for reextraction, sample cleanup, and concentration.

The capabilities of this method were used to resolve a complex CLP environmental sample chromatogram [1] that appeared to have several indistinct and broadly eluting components into a highly resolved elution pattern that contained potentially significant components, separated from the background contamination. In Figure 1 , pollutants that had unique mass spectral features were separated from broad aliphatic hydrocarbon background signals that eluted across about 20 scans (see Figure 1). The procedures were used also to increase the quality of mass spectra selected as a result of inspections of these chromatograms (see subsequent text). Improvements in mass spectral quality were measured in terms of the increase in quality of mass spectral fitted parameters reported by the data system that performed the library search and in achievement of correct identifications of known compounds (e.g., CLP internal standards and surrogates), both in standard mixtures and in environmental samples that also contained unknowns.

The collection of enhanced mass chromatograms was evaluated to determine whether the duration of summing intervals significantly affected the results. Figure $1 \mathrm{a}-\mathrm{d}$ shows the effects of variation of summing intervals from 0.05 to 0.5 scan. Summing intervals in the range of $0.05-0.1$ scan did not provide sufficient integration of individual mass responses for reliable detection of small peaks, such as the one in the range of 7.0-8.0 scans (denoted 1 in Figure 1). By using a scanning interval of 0.33 scans, this peak was readily observable. At the wider summing intervals such as 0.5 scan, closely eluting peaks such as those that occur

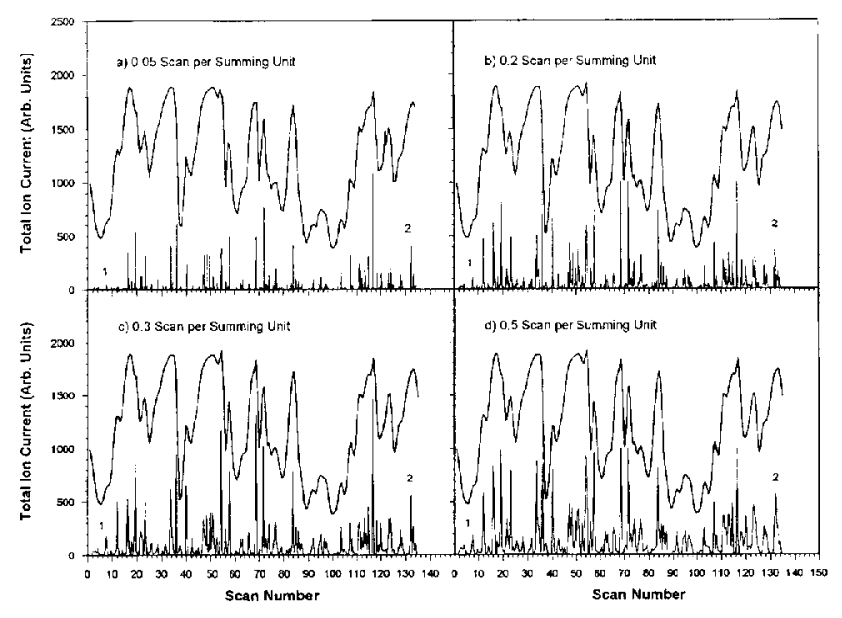

Figure 1. Comparison of native [top traces in graphs (a)-(d)] and enhanced (bottom traces) total ion current chromatograms that demonstrate effects of different summing intervals on the ability to observe and separate the components of the chromatograms. Test peaks 1 and 2 are discussed in the text. 
in the scan range of 131-134 (denoted 2 in Figure 1) were not resolved adequately. These peaks were readily seen to be distinct with a summing interval of 0.1 or 0.2 scans.

Based on these observations, the optimum summing interval for routine applications seemed to be between 0.2 and 0.33 scans. 'I'his hypothesis should be tested for specific applications by using data from the andalysis of standard mixtures to ensure that the chosen summing interval is appropriate for the chromatographic resolution achieved in the analysis. All chromatographic peaks are subsequently treated the same, as long as their peak widths are sufficient to indicate that they are real (not electronic noise, etc.)

With a proper summing interval for the chromatographic resolution achieved by the CLP laboratory, the correct or inherent elution width of a pure compound needed to contain all of the compound's ions was up to approximately 1.5 scans for late-eluting poorly chromatographed components, as demonstrated by the total ion current chromatograms of indeno(1,2,3-cd) pyrene and dibenz $(a, h)$ anthracene shown in Figure 2. In Figure 2, the "incidental peaks" were unidentified conlaminants. In another case, which used a 0.2-scan summing interval, the EXCEL ${ }^{\mathrm{TM}}$ macro successfully baseline-resolved 10 known components (a mixture of chlorinated benzenes and naphthalenes) that eluted within a period of 20 scans. Improved chromatography resulted in shorter correct elution widths of the analytes.

Initial tests of the spectral enhancement algorithm were performed by using CLP mixtures of standards that contained known coeluting compounds to check the technique for accuracy in separation of the spectra of coeluting analytes. The extracted mass spectra were compared to reference spectra contained in the NIST data base. Results of these comparisons showed that usually $80-90 \%$ of the ions in the experimental specIrum that were common to ions in the reference spectra were successfully extracted by using this method. The ions that did not extract well were those with a low ion current and a relatively high noise level. Ions from coeluting interferences, even at much higher con-

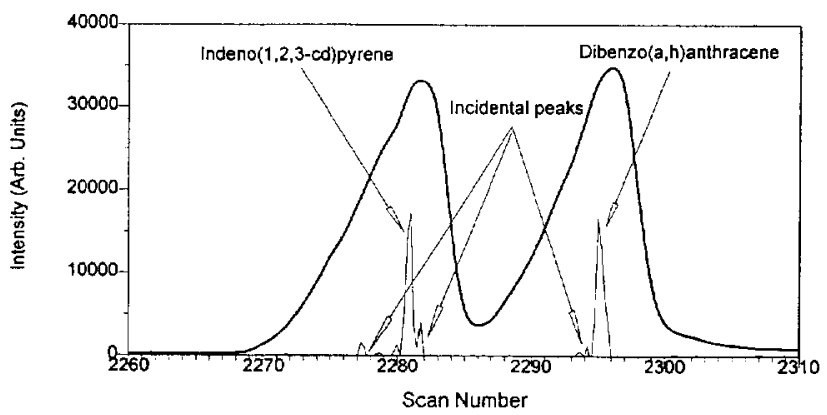

Figure 2. Total ion chromatograms of indeno $(1,2,3-c d)$ pyrene and dibenz $(a, h)$ anthracene that show chromatographic and inherent (enhanced) peak widths of poorly chromatographed lateeluting compounds. centrations, were successfully excluded as long as they did not maximize in the same summing interval and have the same mass as the ions of interest.

A dramatic demonstration of the ability of this method to extract improved mass spectra from a chromatogram with a high background signal is shown in Figure 3 . This example, based on the mass spectrum of naphthalene, showed the value of the spectral enhancement technique for library searches. The enhanced total ion chromatographic peak profile shown in Figure $3 a$ predicted that the mass spectrum was contained in the scan range of 17.2-18.0. The mass spectrum in Figure $3 b$ was obtained from a mass spectral data system by using the standard background subtraction technique that averages the three spectra about the peak maximum and subtracts the average mass spectrum from the two adjoining minima. The HP data system was unable to identify this mass spectrum. The enhanced mass spectrum shown in Figure 3c (scan range 17.2 to 18.0 scans) was correctly identified as naphthalene by the data system, which also produced the reference mass spectrum in Figure $3 \mathrm{~d}$. The failure of the standard procedure appeared to be caused largely by the absence of the masses at $m / z 127$ and 129. The artificial intensity enhancement of the peaks at $m / z 50,51,61-64$, and 74-78 did not hinder the search routine, because it strongly weighted the apparent molecular ion in making identifications. Low-mass responses are common to more potential interfering compounds and thus are more likely to have intensity maxima in the scans under consideration than higher mass interferences. This situation may be the cause of the observed artificially higher intensities of the enhanced lower mass peaks.

Other examples of improved mass spectral quality were provided by the spectral matching quality indicators generated by the HP mass spectral data system for background-subtracted mass spectra directly extracted by the data system against those that were enhanced and extracted by using the spectral enhancement background-rejection technique described in this report. The comparisons shown in Table 2 utilized the data for the mass chromatogram in Figure 1. Both of the examined fitting quality parameters showed small but consistent improvements after the data were subjected to the spectral enhancement procedures. Such small improvements provided significant improvements in mass spectral library search results on solutions of standards [5].

A modification of the spectral enhancement procedure by calculation of the quadratically predicted intensity at each peak was tested. The difference between the calculated apex intensity of any given enhanced peak and the intensity derived from raw data in the native mass chromatogram was less than $2 \%$. This procedure also increased data processing time by about $30 \%$. Ion abundance ratios in CLP data (e.g., for tuning determinations with decafluorotriphenylphos- 

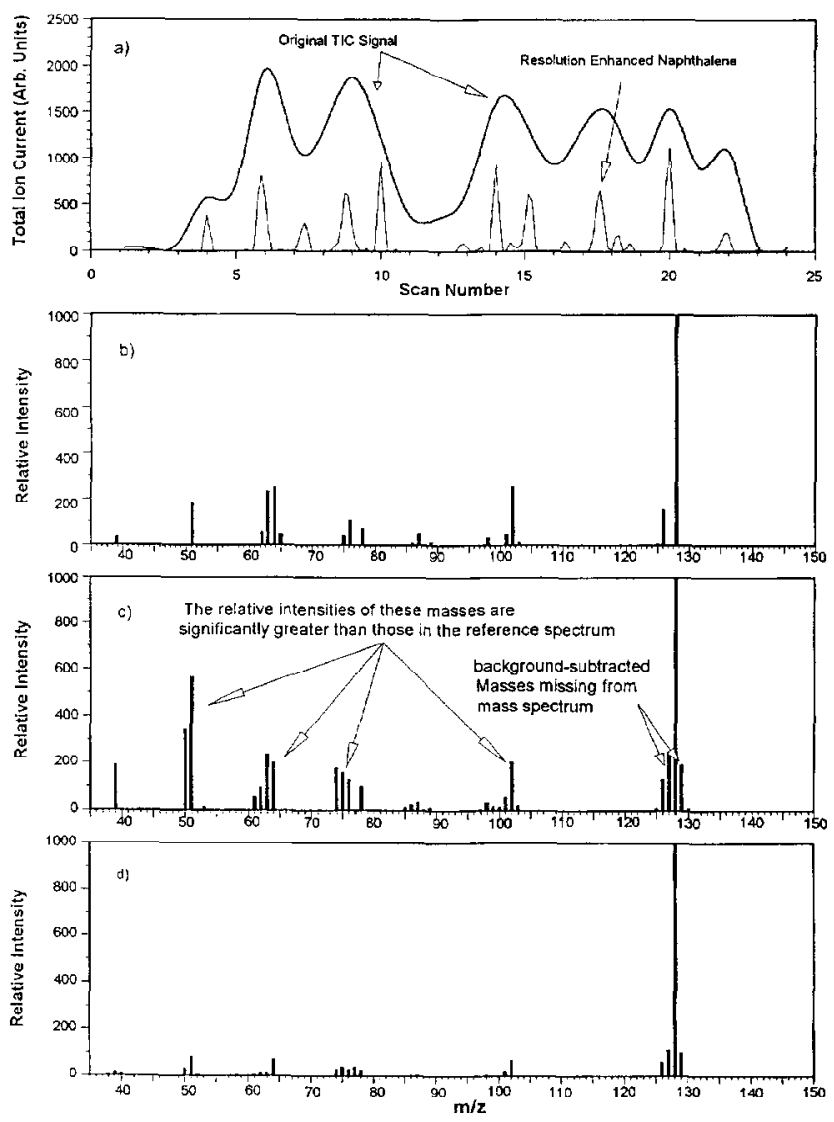

Figure 3. (a) Native and enhanced total ion chromatograms of a naphthalene-containing mixture. (b) Background-subtracted mass spectrum of naphthalene in the mixture retrieved by the HP data system without enhancement. (c) Enhanced mass spectrum of naphthalene in the mixture. (d) NIST reference spectrum.

phine) often vary much more than $2 \%$ among analyses [6], so the improvement was considered to be negligible and not to justify the time requirement. Therefore, this modification to the approach was not studied further.

The stability of the quadratic fitting procedure was tested on 13 different mass chromatographic peaks selected to represent apex offset values $\left(O_{A}\right)$ across the range of \pm 0.45 scan (versus the maximum range of t 0.5 scan). Mass spectrometer noise was simulated by the addition of random noise values in the range \pm 5 , 7.5 , or $10 \%$ of the experimental value to each of the three peak-defining experimental values (scan intensities $I_{L}, I_{O}$, and $I_{R}$ ) and then the quadratic approxima- tion was calculated. These treatments provided the arbitrarily selected total noise versus signal levels of $10,15$, or $20 \%$ peak-to-peak ( $\mathrm{p}-\mathrm{p})$, respectively. These artificially elevated noise levels were created to provide "worst-case scenarios" for actual data quality. The standard deviations obtained from 500 separate random noise trials for each mass chromatographic peak at a given noise level were plotted against the original experimental $\mathcal{O}_{\mathrm{A}}$ value (Figure 4).

A comparison of this three-point exact-fit method with a second order polynomial regression through five points centered on a peak showed that comparable results were obtained about $75 \%$ of the time. However, in the remainder of the cases, the poor signal statistics of the low intensity outlying points caused the fivepoint regression method to predict an optimized retention time for a mass that diverged from those of most masses that belonged to the chromatographic peak. Even in such difficult cases, the three-point method predicted much more convergent optimized retention times. Additionally, standard deviations for regression data generally exceeded those for $O_{A}$ optimized data by a factor of 2 . The three-point exact-fit method was therefore selected as the procedure of choice.

The data showed that for scan-to-scan noise levels up to $20 \% \mathrm{p}^{-\mathrm{p}}$, the quadratically predicted $O_{\Lambda}$ generally varied less than \pm 0.2 scan at the $95 \%$ confidence level (sce Figure 4). This situation indicated that the underlying mass spectral signal maximized within 0.2 scan of the calculated value if the scan-to-scan noise was less than $20 \% \mathrm{p}-\mathrm{p}$. Although scan-to-scan noise information was not readily available for commonly used quadrupole mass spectrometers, it appeared to be unlikely that scan-to-scan noise levels would approach $20 \%$ in properly operating units. This value of 0.2 scan for an approximate $O_{\mathrm{A}}$ uncertainty was about the same as the optimum summing interval. Therefore, a quadratically optimized peak would be calculated to fall within one summing interval of its true position. This situation is acceptable because mass spectral peaks were typically 4-6 summing intervals wide.

The importance of quadratic $O_{A}$ optimization is shown in Figure 5. The original total ion chromatogram is shown in Figure $5 a$ over the enhanced signal obtained by using $O_{S}$ and $O_{A}$ optimization and a summing interval of 0.10 scan. Figure $5 b$ shows this enhanced signal with a 0.25 -scan summing interval. The results obtained by correction for the scan offset $\mathrm{O}_{S}$, but not for the apex offset $\mathrm{O}_{\mathrm{A}}$, are shown in Figure

Table 2. Comparison of native and enhanced HP mass spectral quality indicators

\begin{tabular}{|c|c|c|c|c|c|}
\hline Compound & $\begin{array}{c}\text { Scan number } \\
\text { (Figure 1c) }\end{array}$ & $\begin{array}{l}\text { Native } \\
\text { quality } \\
\text { factor }\end{array}$ & $\begin{array}{c}\text { Enhanced } \\
\text { quality } \\
\text { factor }\end{array}$ & $\begin{array}{l}\text { Native } \\
\text { cross- } \\
\text { correl. }\end{array}$ & $\begin{array}{c}\text { Enhanced } \\
\text { cross- } \\
\text { correl. }\end{array}$ \\
\hline \multicolumn{6}{|l|}{ 2, 5-Dimethyl- } \\
\hline benzo $[b]$ thiophene & 12 & 89 & 91 & 9606 & 9927 \\
\hline Tetradecane & 16 & 89 & 93 & 8462 & 9621 \\
\hline 1 -Ethylnaphthalene & 19 & 96 & 97 & 9904 & 9954 \\
\hline
\end{tabular}




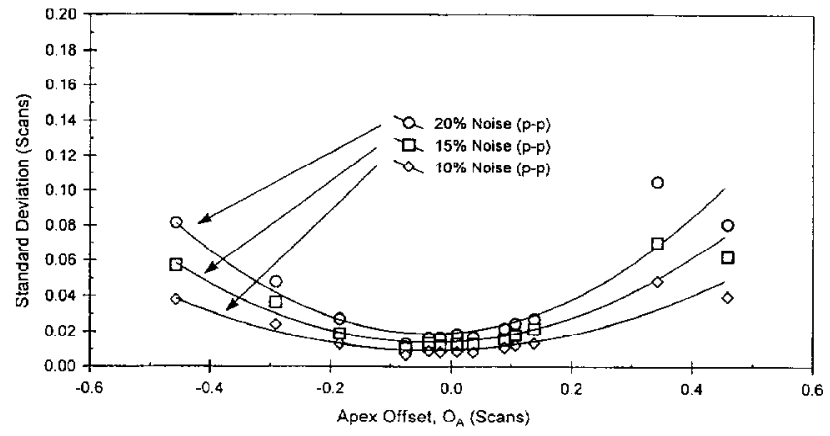

Figure 4. Plot of the standard deviations from 500 measurements of simulated $O_{A}$ values for 13 mass chromatographic peaks under various noise conditions versus the original estimated $O_{A}$ values. The 13 peaks were chosen to cover an $O_{A}$ range of \pm 0.45 scan.

5c, which used a 0.10 -scan summing interval. These data were characterized by a series of peaks of unit scan width, as should be observed because $O_{S}$ is an intrascan effect. The procedure simply identified those ions that maximize in that mass spectrometric scan. The appearance of doublets in most of the peaks was an artifact of the distribution of ion intensities in common CLP mass spectra and the summing interval of 0.10 scan.

Inclusion of the interscan effect $O_{A}$, sharply reduced the number of observable "peaks." The chromatogram in Figure 5a showed potential very narrow peak doublels (labeled 1 and 2 in Figure $5 a$ and b) that were not present in Figure 5b. The appearance of these peak doublets resulted from the short 0.10 -scan summing interval; each of the two "doublets" merged into single peaks when a 0.25 -scan summing interval was used. This occurrence emphasized the importance of selection of the proper sized summing interval.

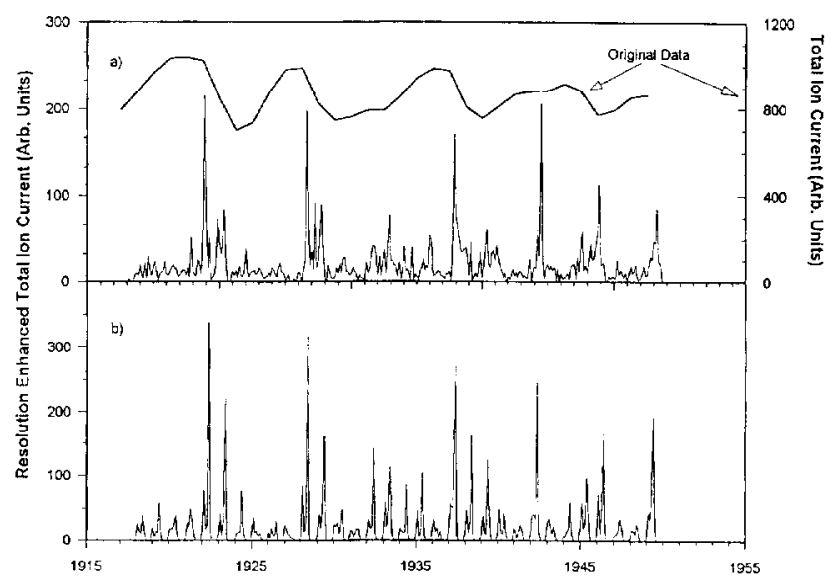

Figure 5. (a) Spectral enhancement results with a 0.10-scan summing interval and $O_{\mathrm{S}}$ (scan offset) and $O_{\mathrm{A}}$ (quadratic fit) optimization (the original unenhanced total ion chromatogram is shown above the enhanced chromatogram). (b) Enhanced chromatogram as in (a) but with a 0.25-scan summing interval. (c) Spectral enhancement results with a 0.10 scan summing interval, without calculation of the quadratic fit $O_{A}$.
Examination of logical neutral losses from observed masses in these two peak doublets was applied to determine whether the corresponding mass responses actually belonged to one peak (compound). This analysis of doublet peaks in Figure 5a indicated that all responses were consistent with the presence of single peaks (compounds), rather than two pairs of closely eluting compounds. An advantage of the simpler $\mathrm{O}_{\mathrm{S}}$ only optimization procedure was the ease of determining whether the selected summing interval was appropriate to group all masses from the same component while maximizing the removal of interference responses. The goal of this $\mathrm{O}_{\mathrm{S}}$-only examination of data was to determine if the mass maxima observed in the scan under investigation were related to both the earlier- and later-eluting adjacent scan numbers or were common with only one of those other two scans. This observation was difficult to make with the full $\mathrm{O}_{\mathrm{S}}+\mathrm{O}_{\mathrm{A}}$ optimization procedure because the combined optimization did not result in cleanly separated adjoining scans.

I'he capabilities of the spectral enhancement concept were ultimately limited by the characteristics of the EXCEL ${ }^{\mathrm{TM}} 5.0$ spreadsheet. The spreadsheet does not permit any sections of a mass chromatogram that contain more than 16,000 records (here, a record comprises a mass, intensity, and retention time) to be addressed. The mass chromatogram was imported, the optimized mass retention times were determined, and the resulting derived mass chromatogram was ohtained in approximately $5-10 \mathrm{~min}$. The resulting summary data such as total ion current mass chromatograms and mass spectra were called derived because they were created from selected subsets of the total data for the run of interest rather than the complete native data set. The derived mass spectrum for each resulting peak of interest was obtained readily in hard copy or electronic format that could be analyzed by a mass spectral data system.

\section{Conclusions}

This approach was found to be effective to extract mass spectra of individual compounds from background signals, which include those of hydrocarbon mixtures that have broad elution profiles on the chromatographic column utilized for the GC/MS acquisitions. This approach could have significant value for the EPA and others who perform analysis of complex samples as a rapid cost-effective alternative to special extract cleanup and reanalysis schemes for removal of chemical interferences that render the mass spectra of selected peaks and/or unknowns difficult or impossible to interpret.

\section{Acknowledgment}

The U.S. Environmental Protection Agency, through its Office of Research and Development, partially funded and collaborated in the research described in this article under contract 68-C0-0049 to 
Lockheed Environmental Systems and Technologies Company. It does not necessarily reflect the views of the EPA or ORD. The U.S. Government has a nonexclusive, royalty-free license in and to any copyright covering this article. Mention of trade names or commercial products does not constitute endorsement or recommendation for use.

\section{References}

1. Statement of Work for Organic Analysis, Multi-Media, MultiConcentration; Contract Laboratory Program, Document Number OLM01.0, L.S. Environmental Protection Agency,
Cincinnati, OH, 1990; including revisions, OLM01.1-OLM03.1; 1990-1994.

2. Test Methods for Determining Solid Waste; SW-846; U.S. Environmental Protection Agency: Washington, D. C., 1992.

3. Colby, B. N. J. Am. Soc. Mass Spectrom. 1992, 3, 558-562.

4. Biller, J. E.; Biemann, K. Anal. Lett. 1974, 7, 515.

5. Colby, B. N.; D'Arcy, P. H. Proceedings of the 11st ASMS Conference of Mass Spectrometry and Allied Topics; San Francisco, CA, 1993; p 813.

6. Donnelly, J. R.; Sovocool, G. W.; Mitchum, R. K. I. Assoc. Off. Anal. Chem. 1988, 71, 434-439. 